\title{
Lymphatic vessels and tertiary lymphoid organs
}

\author{
Nancy H. Ruddle \\ Yale University School of Public Health, New Haven, Connecticut, USA.
}

\begin{abstract}
Tertiary lymphoid organs (TLOs) are accumulations of lymphoid cells in chronic inflammation that resemble LNs in their cellular content and organization, high endothelial venules, and lymphatic vessels (LVs). Although acute inflammation can result in defective LVs, TLO LVs appear to function normally in that they drain fluid and transport cells that respond to chemokines and sphingosine-1-phosphate (S1P) gradients. Molecular regulation of TLO LVs differs from lymphangiogenesis in ontogeny with a dependence on cytokines and hematopoietic cells. Ongoing work to elucidate the function and molecular regulation of LVs in TLOs is providing insight into therapies for conditions as diverse as lymphedema, autoimmunity, and cancer.
\end{abstract}

\section{Introduction}

Lymphatic vessels (LVs) are a series of LYVE1 $1^{+}$PROX1+ vessels throughout the body that function in multiple physiologic and pathophysiologic processes. The initial thin-walled vessels, called capillaries, progress to collecting vessels and then to larger vessels such as the thoracic duct. LVs also express gp38 (podoplanin), VEGFR-2, VEGFR-3, neuropilin-2, angiopoietin-1, and CCL21, although differences in levels of these markers exist between the different vessels. Although other cell types can express some of these markers, none except LVs express the entire range. PROX1 is crucial for development and maintenance of LVs (1); VEGF receptors, especially VEGFR-3, respond to VEGF-C and VEGF-D and induce growth of already existing LVs (2). Fluid is transported through the LVs by means of extrinsic contraction of tissue forces and intrinsic pumping through lymphatic muscle (3). Lymphatic valves prevent backflow and have higher expression of PROX1 than do the cells in the walls of the vessels.

LVs have many functions in homeostasis. They maintain fluid balance, preventing edema by providing drainage of interstitial fluid, provide lipid transport, and serve in an immune capacity by carrying antigen and cells throughout the immune system and regulating this transport through production of chemokines and sphingosine-1-phosphate (S1P) (4). LVs are also found at sites of chronic inflammation, referred to as ectopic or tertiary lymphoid organs (TLOs). This Review considers how closely the functions and regulation of LVs in TLOs resemble those throughout the body, particularly in secondary lymphoid organs (SLOs).

\section{SLOs}

SLOs (LNs, Peyer's patches, spleen, tonsils), which are positioned to provide optimal interactions of components of the immune system with invading pathogens, are remarkably organized with $\mathrm{T}$ and $\mathrm{B}$ cell compartmentalization, APCs, stromal cells, and blood and lymphatic endothelial cells (LECs). Cells are directed to their various locations through the activity of chemokines produced by several different types of stromal cells - fibroblast reticular cells, marginal reticular cells, and endothelial cells (5). Naive cells enter LNs through specialized blood vessels, called high endothelial venules (HEVs), and leave after interaction with antigen, which enters into LNs via LVs. Conduits are very fine microvessels consisting of ECM scaffolding produced by fibroblast reticular cells. They transport low-molecular-weight antigens from the cortex to the para-

Conflict of interest: The author has declared that no conflict of interest exists. Citation for this article: J Clin Invest. 2014;124(3):953-959. doi:10.1172/JCI71611. cortex and into the parenchyma of LNs, where they can contact the HEVs (6). The organization of LNs and their vascular features are presented in Figure 1.

The signals that organize LNs in ontogeny are tightly regulated, which results in the development of individual LNs on a precise temporal and anatomical schedule (7). Members of the lymphotoxin family (LT $\alpha 3$ and LT $\alpha 1 \beta 2$ ) play key roles in generating and maintaining lymphoid organs $(8,9)$ through their production by IL-7R ${ }^{+} \mathrm{CD} 4^{+} \mathrm{CD}^{-}$lymphoid tissue inducer cells. Lymphoid tissue inducer cells interact with cells of mesenchymal origin, called lymphoid tissue organizer cells, which produce chemokines that in turn induce more LT $\alpha \beta$. The role of stromal cells in SLO development and maintenance is becoming better understood (10) as is the response of these cells to neuronal signals, including retinoic acid (11). HEVs are also key organizers of LN development (12), in that they express $\operatorname{LT} \beta R(13)$, respond to $\operatorname{LT} \alpha \beta(14,15)$, and present chemokines and adhesion molecules

\section{Functions of LVs in SLOs}

Afferent LVs bring antigen and APCs to LNs. DCs accumulate in the subcapsular sinus and transmigrate through the floor of that sinus into the $\mathrm{T}$ cell zone, while $\mathrm{T}$ cells access the parenchyma of the LN through the peripheral medullary sinuses (16). Efferent LVs drain activated cells from the LN, which then travel through afferent vessels to the next $\mathrm{LN}$ in the series, into the thoracic duct, and into the bloodstream via the subclavian veins. Markers that distinguish efferent from afferent vessels are not yet known.

Cellular trafficking between the periphery and within LNs is regulated by the lymphoid chemokines CCL19, CCL21, and CXCL13 and by S1P (17). CCL21 is produced or expressed in LNs by stromal cells, HEVs, and LECs. CCR7, the ligand for CCL19 and CCL21, is found on DCs and naive and memory T cells in the blood and is downregulated as they enter into the parenchyma of the LNs. S1P is found in high concentrations in the blood and lymph, where lymphocytes express low levels of the receptor, S1PR1. The receptor is re-expressed at higher levels in the LN, where S1P levels are low. Egress of S1P1R-expressing activated and memory cells from LNs is regulated by the high concentration of S1P in the lymph (ref. 17 and summarized in ref. 18). Thus, LVs play crucial roles in trafficking in LNs via activation and the expression of chemokines and S1P.

Additional potential functions of LVs in LNs include a role in tolerance, as they express self-antigen under the control of AIRE for presentation to T cells (19). LVs may also serve as organizers of LNs in the same manner as HEVs. Conditional inducible knockouts regulated by PROX1 will provide insight into this question. 


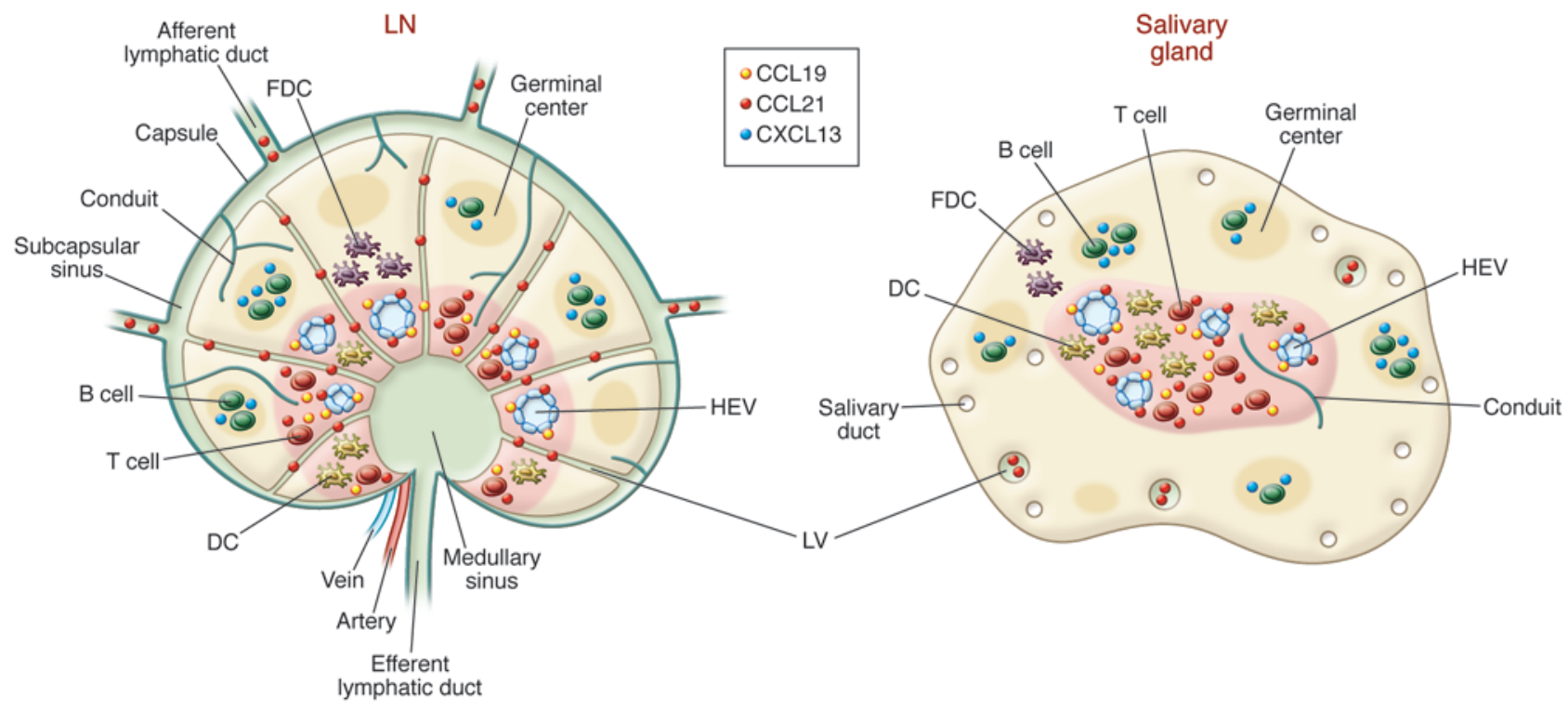

\section{Figure 1}

Comparison of a LN and a salivary gland TLO. Note the presence in both of T and B cell organization, APCs, stromal cells, conduits, chemokines, HEVs, and LVs. One difference is the apparent absence of a capsule in the TLO compared with the LN. Another possible difference concerns uncertainty regarding whether the TLO LVs are afferent and/or efferent. FDC, follicular DC.

\section{TLOs}

TLOs, also referred to as ectopic lymphoid tissues, are accumulations of cells in chronic inflammation (reviewed in ref. 20) and are referred to as tertiary to distinguish them from SLOs. SLOs arise during development at key locations in the body under the control of a precise developmental program, but chronic immune activity in the adult can give rise to similarly organized accumulations of lymphoid cells in almost any non-lymphoid tissue through a process that is not preprogrammed but rather the result of induction by factors in the environment that could elicit the same signals that contribute to LN development (reviewed in refs. 20,21). TLOs are characterized by their cellular, organizational, chemokine, and vascular similarity to SLOs, especially LNs. These similarities include T and B cell compartmentalization, APCs such as DCs and follicular DCs, stromal cells (5), conduits, and a highly organized vascular system of HEVs and LVs (see below) $(5,22,23)$. Common features of TLOs and LNs are depicted in Figure 1.

It has been suggested that TLOs differ from SLOs by the absence of a capsule; however, TLOs in a variety of chronic kidney diseases are in contact with a fibrous capsule (24). The absence of a capsule may reflect the manner in which TLOs arise, compared with LNs. As noted above, the latter arise at predefined locations as a result of stromal and endothelial organizers. Even though most of these elements are also present in TLOs (5), the order in which they populate the TLO may differ from the precise temporal aspect of LNs. The general absence of a capsule may have consequences for trafficking patterns that could differ in TLOs from what is seen in LNs, in which the DCs and T cells migrate through the peripheral medullary sinus to the parenchyma (16).

TLOs arise in several instances of chronic inflammation, including autoimmunity, chronic graft rejection, persistent infection (summarized in ref. 20), atherosclerosis (25), and cancer (26-28). They can be induced experimentally by tissue- specific expression of certain inflammatory mediators (summarized in ref. 20), including members of the lymphotoxin family $(15,29)$, the very cytokines that are crucial for lymphoid organ development and maintenance (8).

As noted above, lymphoid chemokines regulate trafficking of lymphocytes and DCs to and within LNs, and their expression $(20,30)$ is one criterion that defines TLOs. Chemokines play similar roles in LNs and ectopic lymphoid accumulations, since the CCR7-expressing T cells and DCs are situated in the same locality as CCL19 and CCL21 and the CXCR5 ${ }^{+}$B cells are in regions with high CXCL13 levels. TLOs likely function as local sites of antigen presentation and lymphocyte activation, including somatic hypermutation and class switching in B cells (31), which suggests that they facilitate local antimicrobial responses as well as epitope spreading $(32,33)$ and autoimmune exacerbation.

TLOs can progress from a relatively benign to a destructive phase and lose their lymphoid organ characteristics. For example, in the non-obese diabetic (NOD) mouse model of type 1 diabetes mellitus (T1DM), initial pancreatic infiltrates are characterized by HEV development and minimal islet destruction, whereas later stages demonstrate frank islet destruction and diabetes (34). In this model, pancreatic TLOs disappear after removal of the $\beta$ cell antigen and are replaced by tissue fibroblasts. The presence of high proportions of regulatory $\mathrm{T}$ cells in some TLOs $(25,35)$ suggests that immune regulation occurs in these locations.

\section{Functions of LVs in TLOs}

Thin-walled vessels with typical lymphatic markers, including LYVE1 and PROX1, are apparent in the TLOs arising in both clinical settings (autoimmune disease and chronic graft rejection) and experimental models (summarized in ref. 20) (23, 25, 36-40). They are also found in TLOs associated with some tumors (35). The functions of the LVs in TLOs have not been thoroughly investigated but 
are worthy of analysis with regard to fluid balance and transport of antigen and naive, activated, and memory lymphocytes.

Fluid balance is a critical function of LVs in the body. Although production of IL-7 and IL-7R $\alpha$ by afferent LVs is crucial with regard to fluid drainage in the skin during acute inflammation (41), this has not been investigated with regard to the chronic inflammation of TLOs. Since edema is a frequent occurrence in acute inflammation, it is likely that LVs associated with that process serve a drainage function. Lymphangiogenesis accompanies acute inflammation with enhanced lymph flow (42). Seeger and colleagues suggest that inflammation occurs before lymphangiogenesis and gives rise to that process (43). Lymphangiogenesis at early times after immunization or during acute inflammation may be the result of the presence of excess fluid, but the LVs are unable to transport APCs (13) due to defects in lymphatic contraction (44). Thaunat and colleagues have suggested that the existence of edema in an injured tissue results in an insufficient lymphatic outflow that then gives rise to chronic inflammation at the local site (45). They suggest that defective lymphatic drainage is a prerequisite for the development of TLOs and have provided data supporting this concept in chronic graft rejection. Once the TLO has developed, the LVs may function normally and provide fluid balance. Techniques to measure fluid accumulation are available (46) but have not been employed in the local region of a TLO. Future research could take advantage of measurements of interstitial fluid pressure in the local vicinity of a TLO to evaluate whether edema occurs and the LVs are functional. This may be more feasible and meaningful in a clinical setting, in which a relatively accessible TLO such as the joint in rheumatoid arthritis (RA) provides a logical study site.

Do TLO LVs transport soluble or cell-associated antigen as they do in LNs? Perhaps this is a moot point, because in contrast to SLOs, the antigen is continuously locally available in TLOs. For example, proteins such as insulin in the pancreatic islet are in immediate proximity to or even a part of the TLO in T1DM. Thus, the necessity for antigen transport to the TLO through an extensive LV system might not be necessary, and the LVs may not serve that function. The presentation of self-antigen in LNs (19) that has been suggested as a mechanism for self-tolerance has not been investigated in TLOs and is an important area for future research.

LVs transport cells to primary LNs and then to downstream LNs. Is there such a cellular transport function for TLO-associated LVs, in which cells travel to and from the LNs? LVs that are packed with lymphocytes are prominent in some TLOs (ref. 36 and Figure 2), which suggests that they do transport lymphocytes. Additional evidence supporting an afferent function for TLO LVs comes from the study of myasthenia gravis (MG), an autoimmune disease characterized by thymic TLOs with HEVs and B cell follicles. HEVs are the normal entry points for lymphocytes from the circulation into the LN parenchyma, but in this particular case, the HEVs are negative for CCL21, suggesting that it could be difficult for CCR7 ${ }^{+}$ cells to enter through their usual (HEV) route. In most humans, thymic LVs do not normally express CCL21, but in patients with MG, thymic PROX $1^{+}$VEGFR- $3^{+}$vessels do express this chemokine (47). Because B and T cells in this instance express CCR7 and naive $B$ cells migrate across a CCL21 gradient, the authors suggest that although the thymus does not normally contain afferent LVs, in this pathologic TLO, the LVs function in that capacity and contribute to thymic hyperplasia. In the early stages of non-obese diabetes, the infiltrates exhibit the characteristics of TLOs, includ- ing HEVs and LVs $(23,48,49)$, T and B cells, and macrophages. CCL21 is expressed on such LVs and is reduced upon treatment with complete Freund's adjuvant (49), as is the lymphocytic infiltration, which again suggests that the CCL21-expressing LVs act as afferent vessels into the TLOs.

TLO-associated LVs may also function in an efferent capacity by transporting activated lymphocytes to the periphery. CCL21 is a crucial chemokine for the traffic of LV-resident DCs to LNs; therefore, the presence of CCL $21^{+} \mathrm{LV}$ s in TLOs would support the idea that they can serve to transport CCR7 ${ }^{+}$DCs and lymphocytes. Indeed, there are several examples of CCL21 expression by TLO-associated LVs in humans with RA, Crohn's disease, Sjögren's syndrome, lichen planus (50), and chronic allograft rejection (51) and in the TLOs of pancreatic infiltrates in NOD (23) and RIPLT $\alpha$ mice (30).

Support for the idea that LVs in TLOs serve an efferent function comes from the observation that continual administration of FTY720, an S1P receptor agonist that blocks lymphocyte egress from LNs (52), prevents diabetes in NOD mice (53). This is only effective if the mice have already developed pancreatic TLOs (23). Treatment results in additional accumulation of lymphocytes in the pancreatic TLOs, which is reversed upon cessation, resulting in rapid islet destruction and diabetes $(23,54)$. These data suggest that lymphocyte trafficking through LVs in TLOs in NOD mice occurs under the control of the lymph S1P gradient and re-expression of its receptor by $\mathrm{T}$ cells, as occurs in LNs. These data are also consistent with the observation that FTY720 also prevents egress from inflamed tissues into afferent lymphatics $(55,56)$ and raise the exciting possibility that inhibitors of LV function could prevent diabetes and other autoimmune diseases systemically by preventing trafficking from the TLO to the LNs. It is not known whether the LVs in TLOs produce S1P as they do in the rest of the body (4).

\section{Regulation of LVs in TLOs}

During ontogeny, LVs develop after the embryonic blood vessels have formed, sprouting from the cardinal vein (57). These events are orchestrated by products of the homeobox genes (SOX18 and PROX1) and growth factors and their receptors (VEGF-C and VEGFR-3) and require platelets (ref. 58 and reviewed in ref. 59). The involvement of additional cell types is suggested by the existence of lymphangioblasts, which are distinct from blood endothelial cells, in developing tadpoles $(60)$. Studies in chickens $(61,62)$ and mice (63-65) support a role for mesodermal cells that express macrophage and lymphatic markers that become, integrate into, or support LVs through their production of VEGFs.

Regulation of lymphangiogenesis in TLOs is poorly understood. It is not known whether LVs precede the cellular accumulations and organization into TLOs. Since LVs produce the lymphoid chemokine CCL21, it is possible that they are among the stromal organizers of LNs and TLOs. This is consistent with the role that HEVs play in LNs (12) and presumably in TLOs, since HEVs are similarly, if not identically, regulated in those sites (15). Data from doxycycline-induced TLOs in RIPLT $\alpha$ mice indicate that the presence of LVs is a very early event that can occur prior to apparent leukocytic infiltration into the affected organ (37) and that occurs in the absence of obvious edema. Angiogenesis occurs in inflammation and platelets are present, which indicates that the important players in embryonic lymphangiogenesis may participate. Thus, the possibility, though remote, exists that a recapitulation of the developmental program could occur - that is, that LVs in 

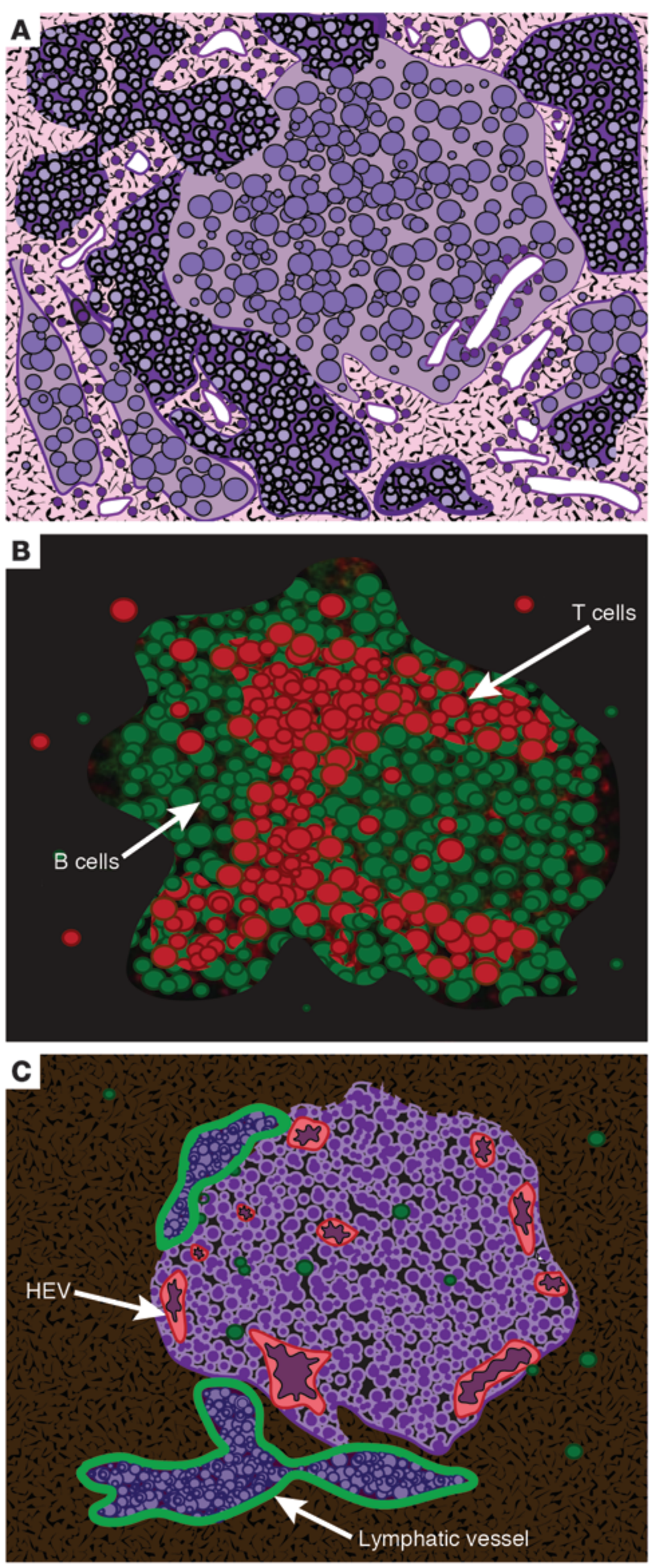

inflammation could arise from veins as they do in development. The presence of vessels that express markers of HEVs and LVs in the inflamed LN supports this scenario (13). On the other hand, the presence of blood vessels and their nearby LVs in TLOs suggests that lymphangiogenesis in inflammation occurs by sprouting from existing LVs. But what are the cells that signal these events? DCs, macrophages, and T and B cells have been implicated

\section{Figure 2}

Diagrammatic rendering of actual staining of a TLO from a mouse salivary gland. (A) Giemsa staining of TLO reveals the presence of leukocytes. (B) Staining for B cells (B220, green) and T cells (CD3, red). (C) Staining for HEVs (MECA 79, red) and LVs (LYVE1, green). Note that the LVs are filled with lymphocytes, which indicates that transport of these cells is occurring and, thus, suggests that these LVs possess afferent and/or efferent function. Adapted from Proceedings of the National Academy of Sciences of the United States of America (36); copyright (2007) National Academy of Sciences, USA.

in the regulation of LVs in acute inflammation (13, 66-68). More recently, neutrophils have also been proposed to increase bioavailability of VEGF-A and to produce VEGF-D (69). Different cells may be important at different times in various tissues. For example, B cells appear to be important in the lymphangiogenesis that occurs in LNs during inflammation, but only at the early stages after immunization $(13,69)$.

The participation of macrophages in lymphangiogenesis in acute inflammation has been well documented, although the precise nature of their role is a subject of considerable controversy. Data in a model of corneal transplant lymphangiogenesis suggest that macrophages can actually transdifferentiate into LECs (70); that is, that macrophages themselves are precursors to LECs $(61,62,64,71)$. The expression of LYVE1 by macrophages could be interpreted as evidence that this is the case. On the other hand, the expression of this marker on both cell types may be a red herring. The presence of additional LV markers such as PROX1 would go some way toward resolving this issue.

The well-known capacity of macrophages to produce growth factors, such as VEGF-C, VEGF-A, VEGF-D, PDGF, and adrenomedullin (summarized in ref. 72) suggests that it is likely that these cells contribute indirectly. Macrophages produce TNF- $\alpha$, which has been implicated in lymphangiogenesis in a model of acute Mycoplasma pulmonis lung infection (73), which also supports an indirect mechanism for the functions of macrophages in LVs rather than direct transdifferentiation. Kerjaschki and colleagues demonstrated the presence of host bone marrow-derived precursors in association with LVs in the TLOs of chronically rejecting kidneys (39). Osteoclast precursors, which include cells with macrophage properties, participate in lymphangiogenesis in a model of TNF transgene- and serum-mediated RA (74). During low-dose, streptozotocin-induced pancreatic inflammation, there is a marked increase in macrophages in and around the islets. One population produces VEGF-C, and another LYVE1+ ${ }^{+}$population appears to integrate or differentiate into LECs (75). As in ontogeny or acute inflammation, it is unclear whether macrophages differentiate into LECs, integrate into LVs, and/or produce growth factors in TLOs. Lineage-tracing experiments might resolve this controversy.

Cytokines contribute to lymphangiogenesis in acute inflammation, although there have been few studies evaluating their roles in the chronic inflammation-associated TLOs. The question of LT participation is of particular interest given both its crucial role in lymphoid organ development and its ability to induce TLOs. LT $\alpha$ appears to play a minor, but real, role in lymphangiogenesis in development, as $\mathrm{Lta}^{-/-}$mice exhibit somewhat diminished LV function (37). Both LT $\alpha$ and TNF- $\alpha$ contribute to lymphangiogenesis in acute inflammation in M. pulmonis infection $(37,73)$. LT $\alpha$ also participates in acute lymphangiogenesis in the skin (37). LVs are induced in TLOs of RIPLT $\alpha$ mice. In this case, lymphangiogenesis 
occurs in the absence of LT $\beta$ (37). On the other hand, in a model of CXCL13-induced thyroid TLO, LVs are inhibited by treatment with LT $\beta$ R-Ig (38), implicating the LT $\alpha \beta$ complex. Further experimentation in spontaneous TLOs, such as Sjögren's syndrome, RA, and T1DM may reveal which cytokines, in addition to the TNF family, contribute to this process, whether the classical or alternative NF-KB pathways are involved, and whether these effects directly modulate LVs and/or are mediated indirectly through their induction of infiltrating cells that produce growth factors such as VEGF-C or VEGF-D.

The plasticity of LVs is a reflection of their environment, which influences their function, especially in the case of inflammation. CCL21 is particularly sensitive to inflammation. The low level of CCL21 protein on mouse dermal LVs is increased in explanted TNF- $\alpha$-treated skin or oxazolone-induced contact sensitivity (76). TNF- $\alpha$ treatment of human dermal LECs results in increased CCL21 mRNA transcription, translation, and cell surface protein expression (76). As noted above, CCL21 expression becomes detectable on LVs in the thymus of TLOs in patients with MG (47). Immunofluorescence and microarray studies that compare LECs from acutely inflamed and resting mouse skin reveal increased expression of CCL21 and several other inflammatory genes (77). Interestingly, there is downregulation of other genes, including Vegfr 3 and Prox1. Extension of these studies to LVs in TLOs may reveal differences due to the chronic nature of stimulation, and data from a mouse corneal model of recurrent inflammation suggest that this is the case (78). The authors suggest that, in chronic inflammation, LVs retain memory in their accelerated development of a network of functional LVs (78). The ability to isolate LVs on the basis of their antigen expression (77) or transgene induced fluorescence (79) will allow their molecular analysis and comparison to vessels from resting LNs, activated LNs, and TLOs.

\section{Future research directions}

The functions of LVs in TLOs may be revealed by a detailed molecular signature. Are there are differences in gene expression between afferent and efferent LVs in LNs, and is this reflected in different LVs in TLOs? As noted above, new techniques allowing isolation, purification, and single-cell in situ analysis will provide the tools for determination of gene expression. Are there any genes that are differentially expressed in LVs from TLOs compared with those in the rest of the body? If so, it will be possible to preferentially affect those vessels with inhibitors of their function while leaving remaining LVs intact.

Analysis of lymphocyte, DC, and antigen-trafficking patterns in TLOs in real time in vivo is now possible with the use of mice that express fluorescent markers for HEVs $(80)$ and LVs $(79,81)$. Although the technique of in vivo imaging is well established for analysis of trafficking in LNs (82), addressing this issue in TLOs is a greater challenge. The key is to study TLOs in tissues that are amenable to these techniques, which will allow the evaluation of questions concerning LV insufficiency, memory, and plasticity in LVs in TLOs, and on a functional level, to determine whether valves and muscles occur in these vessels. The analysis of TLOs in mice whose LVs are conditionally deleted or induced through PROX1 regulation will provide information regarding the importance of fluid drainage functions of LVs in this context. Finally, the influence of the local environment on the LVs in TLOs in different organs must be analyzed. How do differences the local milieu of the pancreas compared with that of the digestive tract, salivary gland, or joint influence the LVs that arise in the TLOs in those organs during T1DM, inflammatory bowel disease, Sjögren's syndrome, or RA?

\section{Clinical implications}

Elucidation of LV regulatory mechanisms in TLOs will contribute to the development of therapies to either promote or inhibit their formation, but we must keep in mind that LVs may be beneficial or detrimental depending on their context. The following sections discuss the implications of LV modulation in autoimmunity, cancer, and fluid balance/lymphedema.

Autoimmunity. Inhibition of TLO LVs may be beneficial in autoimmunity, since they contribute to exacerbation by epitope spreading; this could occur through their transport of naive lymphocytes and APCs to the local site. Treatments that discourage LV development, such as inhibition of macrophages (clodronate), cytokines (antibodies or receptor blockers), and growth factors (e.g., VEGFs) or those agents that inhibit function, such as the S1P1 receptor agonist FTY720, could be beneficial. The identification of markers preferentially expressed or expressed at a high level on TLO LVs would allow for their preferential inhibition. Inhibition of LV function might be protective in MG. LVs most likely contribute to priming by bringing naive lymphocytes into the thymic TLOs, thus leading to sensitization to the nicotinic AChR and pathogenic antibodies to that antigen.

One must be cautious about the possibility that wide-scale inhibition of LVs might result in disastrous lymphedema. Thus, the goal should be targeted delivery to the TLO by vehicles such as nanoparticles $(83,84)$ that could deliver LV inhibitors (e.g., VEGFR-3-targeted antibodies) at the selected site. One can envision induced temporary perturbations of LV function while leaving the main beneficial functions intact. This will be possible as more information becomes available regarding the special properties and regulation of LVs in TLOs.

Cancer. Lymphangiogenesis occurs in many human tumors (summarized in ref. 85), and an enormous literature emphasizes a detrimental role for LVs as contributors to metastasis. A logical approach to inhibiting this spread would be the use of lymphangiogenesis inhibitors (85). A summary of the actual agents that are in clinical use for this purpose is presented in a recent review article (86). As noted above, the hazards of such approaches can lead to unintended lymphedema.

It may be time to rethink the approach of inhibiting LVs in cancer, as they may contribute to defense in some cancers. TLOs are clearly beneficial in some cancers, particularly if there is a generous component of HEVs (28). The interpretation of these data is that the TLO serves as a site of priming of naïve cells that enter via the HEVs and become sensitized to the tumor antigens. Since the high number of HEVs also correlates with reduced metastases, we need to consider that the response generated in the tumor is effective elsewhere. Perhaps this occurs because memory cells leave the tumor TLO via LVs, and the activated cells traffic throughout the body and seek out metastatic disease. Thus, in this context, the LVs in tumor TLOs are beneficial, as they serve as an exit for activated lymphocytes. It might be of benefit to encourage the development of LVs in this context (28).

Lymphedema. Engineering artificial TLOs may be of benefit in counteracting the effects of lymphedema. LVs in TLOs most likely contribute to fluid balance; understanding the roles of cytokines and cells in LVs in TLOs could be advantageous in efforts to 
encourage lymphangiogenesis and fluid drainage in situations where the LVs are defective. It has been estimated that 140-250 million people worldwide suffer from this painful, disfiguring problem, which can be the result of an anatomical abnormality of LVs of genetic origin (primary lymphedema) or the result of damage or obstruction of LVs, due to cancer, cancer treatment (surgery or radiation), or infection (secondary lymphedema). The use of inflammation-induced LVs might be beneficial in these situations. However, as noted above, the lymphangiogenesis that initially occurs in acute inflammation can result in defective LVs (44); therefore, it is crucial to understand the factors that generate and maintain functional, mature, LVs.

\section{Conclusions}

The secrets of LVs are being rapidly revealed, but there is much to be learned. Their existence in TLOs adds to the complexity and excitement in this emerging field. Major questions remain concerning LVs in general and in TLOs in particular. The presence of LVs in TLOs has been well documented, and evidence for their functions has been explored. Methods, particularly imaging techniques, to further probe the functions of LVs in LNs and TLOs are available and continually improving. The regulation of lymphangiogenesis in TLOs remains a fertile area of research and is of key importance for the prophylaxis and treatment of autoimmunity, cancer, and lymphedema.

\section{Acknowledgments}

Work in the author's laboratory was supported by NIH grants R21HL098711 and U19-AI082713 and by JDRF grant 4-2007-1059.

Address correspondence to: Nancy H. Ruddle, Department of Epidemiology of Microbial Diseases, Yale University School of Public Health, 60 College St., New Haven, Connecticut 06510, USA. Phone: 203.785.3281; Fax: 203.737.2921; E-mail: nancy.ruddle@yale.edu.
1. Wigle JT, Oliver G. Prox1 function is required for the development of the murine lymphatic system. Cell. 1999;98(6):769-778.

2. Alitalo K. The lymphatic vasculature in disease. Nat Med. 2011;17(11):1371-1380.

3. Zawiega D. Contractile physiology of lymphatics. Lymphat Res Biol. 2009;7(2):87-96.

4. Pham TH, et al. Lymphatic endothelial cell sphingosine kinase activity is required for lymphocyte egress and lymphatic patterning. J Exp Med. 2010;207(1):17-27.

5. Stranford S, Ruddle NH. Follicular dendritic cells, conduits, lymphatic vessels, and high endothelial venules in tertiary lymphoid organs: parallels with lymph node stroma. Front Immunol. 2012;3:350.

6. Sixt M, et al. The conduit system transports soluble antigens from the afferent lymph to resident dendritic cells in the $\mathrm{T}$ cell area of the lymph node. Immunity. 2005;22(1):19-29.

7. Rennert PD, James D, Mackay F, Browning JL, Hochman PS. Lymph node genesis is induced by signaling through the lymphotoxin $\beta$ receptor. Immunity. 1998;9(1):71-79.

8. Ruddle NH, Akirav EM. Secondary lymphoid organs: responding to genetic and environmental cues in ontogeny and the immune response. J Immunol. 2009;183(4):2205-2212.

9. Koni PA, Sacca R, Lawton P, Browning JL, Ruddle $\mathrm{NH}$, Flavell RA. Distinct roles in lymphoid organogenesis for lymphotoxins $\alpha$ and $\beta$ revealed in lymphotoxin $\beta$-deficient mice. Immunity. 1997;6(4):491-500.

10. Kain MJ, Owens BM. Stromal cell regulation of homeostatic and inflammatory lymphoid organogenesis. Immunology. 2013;140(1):12-21.

11. van de Pavert SA, Mebius RE. New insights into the development of lymphoid tissues. Nat Rev Immunol. 2010;10(9):664-674.

12. Onder L, et al. Endothelial cell-specific lymphotoxin $\beta$ receptor signaling is critical for lymph node and high endothelial venule formation. J Exp Med. 2013;210(3):465-473

13. Liao S, Ruddle NH. Synchrony of high endothelial venules and lymphatic vessels revealed by immunization. J Immunol. 2006;177(5):3369-3379.

14 . Browning JL, et al. Lymphotoxin- $\beta$ receptor signaling is required for the homeostatic control of HEV differentiation and function. Immunity. 2005;23(5):539-550.

15. Drayton DL, Ying X, Lee J, Lesslauer W, Ruddle NH. Ectopic LT $\alpha \beta$ directs lymphoid organ neogenesis with concomitant expression of peripheral node addressin and a HEV-restricted sulfotransferase. J Exp Med. 2003;197(9):1153-1163.

16. Braun A, et al. Afferent lymph-derived T cells and DCs use different chemokine receptor
CCR7-dependent routes for entry into the lymph node and intranodal migration. Nat Immunol. 2011;12(9):879-887.

17. Cyster JG, Schwab SR. Sphingosine-1-phosphate and lymphocyte egress from lymphoid organs. Annu Rev Immunol. 2012;30:69-94.

18. Girard JP, Moussion C, Förster R. HEVs, lymphatics and homeostatic immune cell trafficking in lymph nodes. Nat Rev Immunol. 2012;12(11):762-773.

19. Cohen JN, et al. Lymph node-resident lymphatic endothelial cells mediate peripheral tolerance via Aire-independent direct antigen presentation. J Exp Med. 2010;207(4):681-688.

20. Drayton DL, Liao S, Mounzer RH, Ruddle NH. Lymphoid organ development: from ontogeny to neogenesis. Nat Immunol. 2006;7(4):344-353.

21. Eberl G. From induced to programmed lymphoid tissues: the long road to preempt pathogens. Trends Immunol. 2007;28(10):423-428.

22. Link A, et al. Association of T-zone reticular networks and conduits with ectopic lymphoid tissues in mice and humans. Am J Pathol. 2011; 178(4):1662-1675.

23. Penaranda C, Tang Q, Ruddle NH, Bluestone JA. Prevention of diabetes by FTY720-mediated stabilization of peri-islet tertiary lymphoid organs. Diabetes. 2010;59(6):1461-1468.

24. Mandache E, Penescu M. Renal subcapsular tertiary lymphoid aggregates in chronic kidney diseases. Rom J Morphol Embryol. 2011;52(4):1219-1225.

25 . Gräbner R, et al. Lymphotoxin $\beta$ receptor signaling promotes tertiary lymphoid organogenesis in the aorta adventitia of aged ApoE ${ }^{-/}$mice. J Exp Med. 2009;206(1):233-248.

26. Cipponi A, et al. Neogenesis of lymphoid structures and antibody responses occur in human melanoma metastases. Cancer Res. 2012;72(16):3997-4007.

27. Bergomas F, et al. Tertiary intratumor lymphoid tissue in colo-rectal cancer. Cancers (Basel). 2011; $4(1): 1-10$.

28. Martinet $\mathrm{L}$, et al. Human solid tumors contain high endothelial venules: association with T- and B-lymphocyte infiltration and favorable prognosis in breast cancer. Cancer Res. 2011;71(17):5678-5687.

29. Kratz A, Campos-Neto A, Hanson MS, Ruddle NH. Chronic inflammation caused by lymphotoxin is lymphoid neogenesis. J Exp Med. 1996; 183(4):1461-1472.

30. Hjelmström P, Fjell J, Nakagawa T, Sacca R, Cuff CA, Ruddle NH. Lymphoid tissue homing chemokines are expressed in chronic inflammation. Am J Pathol. 2000;156(4):1133-1138.

31. Schröder AE, Greiner A, Seyfert C, Berek C. Differentiation of $\mathrm{B}$ cells in the nonlymphoid tissue of the synovial membrane of patients with rheu- matoid arthritis. Proc Natl Acad Sci U S A. 1996; 93(1):221-225.

32. McMahon EJ, Bailey SL, Castenada CV, Waldner $\mathrm{H}$, Miller SD. Epitope spreading initiates in the CNS in two mouse models of multiple sclerosis. Nat Med. 2005;11(3):335-339.

33. Kuerten S, et al. Tertiary lymphoid organ development coincides with determinant spreading of the myelin-specific T cell response. Acta Neuropathol. 2012;124(6):861-873.

34. André I, Gonzalez A, Wang B, Katz J, Benoist C, Mathis D. Checkpoints in the progression of autoimmune disease: lessons from diabetes models. Proc Natl Acad Sci US A. 1996;93(6):2260-2263.

35. Martinet L, Filleron T, Le Guellec S, Rochaix P, Garrido I, Girard JP. High endothelial venule blood vessels for tumor-infiltrating lymphocytes are associated with lymphotoxin $\beta$-producing dendritic cells in human breast cancer. J Immunol. 2013;191(4):2001-2008.

36. Liao S, Bentley K, Lebrun M, Lesslauer W, Ruddle FH, Ruddle NH. Transgenic LacZ under control of Hec-6st regulatory sequences recapitulates endogenous gene expression on high endothelial venules. Proc Natl Acad Sci U S A. 2007;104(11):4577-4582.

37. Mounzer RH, et al. Lymphotoxin- $\alpha$ contributes to lymphangiogenesis. Blood. 2010;116(12):2173-2182.

38. Furtado GC, et al. Lymphotoxin $\beta$ receptor signaling is required for inflammatory lymphangiogenesis in the thyroid. Proc Natl Acad Sci U S A. 2007;104(12):5026-5031.

39. Kerjaschki D, et al. Lymphatic neoangiogenesis in human kidney transplants is associated with immunologically active lymphocytic infiltrates. J Am Soc Nephrol. 2004;15(3):603-612.

40. Kerjaschki D, et al. Lymphatic endothelial progenitor cells contribute to de novo lymphangiogenesis in human renal transplants. Nat Med. 2006;12(2):230-234.

41. Iolyeva $M$, et al. Interleukin-7 is produced by afferent lymphatic vessels and supports lymphatic drainage. Blood. 2013;122(13):2271-2281.

42. Kataru RP, et al. Critical role of CD $11 b+$ macrophages and VEGF in inflammatory lymphangiogenesis, antigen clearance, and inflammation resolution. Blood. 2009;113(22):5650-5659.

43. Seeger H, Bonani M, Segerer S. The role of lymphatics in renal inflammation. Nephrol Dial Transplant. 2012;27(7):2634-2641.

44. Liao S, et al. Impaired lymphatic contraction associated with immunosuppression. Proc Natl Acad Sci US A. 2011;108(46):18784-18789.

45. Thaunat O, Kerjaschki D, Nicoletti A. Is defective lymphatic drainage a trigger for lymphoid neogenesis? Trends Immunol. 2006;27(10):441-445. 
46. Karlsen TV, Karkkainen MJ, Alitalo K, Wiig H. Transcapillary fluid balance consequences of missing initial lymphatics studied in a mouse model of primary lymphoedema. J Physiol. 2006;574(pt 2):583-596.

47. Berrih-Aknin S, et al. CCL21 overexpressed on lymphatic vessels drives thymic hyperplasia in myasthenia. Ann Neurol. 2009;66(4):521-531.

48. Bistrup A, et al. Detection of a sulfotransferase (HEC-GlcNAc6ST) in high endothelial venules of lymph nodes and in high endothelial venule-like vessels within ectopic lymphoid aggregates: relationship to the MECA-79 epitope. Am J Pathol. 2004; 164(5):1635-1644

49. Qu P, Ji RC, Shimoda H, Miura M, Kato S. Study on pancreatic lymphatics in nonobese diabetic mouse with prevention of insulitis and diabetes by adjuvant immunotherapy. Anat Rec A Discov Mol Cell Evol Biol. 2004;281(2):1326-1336.

50. Manzo A, et al. CCL21 expression pattern of human secondary lymphoid organ stroma is conserved in inflammatory lesions with lymphoid neogenesis. Am J Pathol. 2007;171(5):1549-1562.

51. Nykänen AI, et al. Targeting lymphatic vessel activation and CCL 21 production by vascular endothelial growth factor receptor-3 inhibition has novel immunomodulatory and antiarteriosclerotic effects in cardiac allografts. Circulation. 2010; 121(12):1413-1422.

52. Mandala S, et al. Alteration of lymphocyte trafficking by sphingosine-1-phosphate receptor agonists. Science. 2002;296(5566):346-349.

53. Maki T, Gottschalk R, Monaco AP. Prevention of autoimmune diabetes by FTY720 in nonobese diabetic mice. Transplantation. 2002;74(12):1684-1686.

54. Maki T, Gottschalk R, Ogawa N, Monaco AP. Prevention and cure of autoimmune diabetes in nonobese diabetic mice by continuous administration of FTY720. Transplantation. 2005;79(9):1051-1055.

55. Ledgerwood LG, et al. The sphingosine 1-phosphate receptor 1 causes tissue retention by inhibiting the entry of peripheral tissue T lymphocytes into afferent lymphatics. Nat Immunol. 2008; 9(1):42-53

56. Brown $\mathrm{MN}$, et al. Chemoattractant receptors and lymphocyte egress from extralymphoid tissue: changing requirements during the course of inflammation. J Immunol. 2010;185(8):4873-4882.

57. Srinivasan RS, et al. Lineage tracing demonstrates the venous origin of the mammalian lymphatic vasculature. Genes Dev. 2007;21(19):2422-2432.

58. Abtahian F, et al. Regulation of blood and lym- phatic vascular separation by signaling proteins SLP-76 and Syk. Science. 2003;299(5604):247-251.

59. Tammela T, Alitalo K. Lymphangiogenesis: molecular mechanisms and future promise. Cell. 2010;140(4):460-476.

60. Ny A, et al. A genetic Xenopus laevis tadpole model to study lymphangiogenesis. Nat Med. 2005; 11(9):998-1004

61. Wilting J, et al. Dual origin of avian lymphatics. Dev Biol. 2006;292(1):165-173.

62. Wilting J, et al. Development of the avian lymphatic system. Microsc Res Tech. 2001;55(2):81-91.

63. Buttler K, et al. De novo hem- and lymphangiogenesis by endothelial progenitor and mesenchymal stem cells in immunocompetent mice [published online ahead of print September 1, 2013]. Cell Mol Life Sci. doi:10.1007/s00018.013.1460-8.

64. Buttler K, Ezaki T, Wilting J. Proliferating mesodermal cells in murine embryos exhibiting macrophage and lymphendothelial characteristics. BMC Dev Biol. 2008;8:43.

65. Buttler K, et al. Mesenchymal cells with leukocyte and lymphendothelial characteristics in murine embryos. Dev Dyn. 2006;235(6):1554-1562.

66. Chyou $\mathrm{S}$, et al. Coordinated regulation of lymph node vascular-stromal growth first by $\mathrm{CD} 11 \mathrm{c}^{+}$ cells and then by T and B cells. J Immunol. 2011; 187(11):5558-5567.

67. Angeli V, et al. B cell-driven lymphangiogenesis in inflamed lymph nodes enhances dendritic cell mobilization. Immunity. 2006;24(2):203-215.

68. Muniz LR, Pacer ME, Lira SA, Furtado GC. A critical role for dendritic cells in the formation of lymphatic vessels within tertiary lymphoid structures. J Immunol. 2011;187(2):828-834.

69. Tan KW, et al. Neutrophils contribute to inflammatory lymphangiogenesis by increasing VEGF-A bioavailability and secreting VEGF-D. Blood. 2013; 122(22):3666-3677.

70. Maruyama K, et al. Inflammation-induced lymphangiogenesis in the cornea arises from CD11b-positive macrophages. J Clin Invest. 2005; 115(9):2363-2372.

71. Wilting J, Becker J, Buttler K, Weich HA. Lymphatics and inflammation. Curr Med Chem. 2009; 16(34):4581-4592.

72. Ran S, Montgomery KE. Macrophage-mediated lymphangiogenesis: the emerging role of macrophages as lymphatic endothelial progenitors. Cancers (Basel). 2012;4(3):618-657.

73. Baluk P, et al. TNF- $\alpha$ drives remodeling of blood ves- sels and lymphatics in sustained airway inflammation in mice. J Clin Invest. 2009;119(10):2954-2964.

74. Zhang Q, et al. Increased lymphangiogenesis in joints of mice with inflammatory arthritis. Arthritis Res Ther. 2007;9(6):R118.

75. Yin $\mathrm{N}$, et al. Lymphangiogenesis is required for pancreatic islet inflammation and diabetes. PLoS One. 2011;6(11):e28023.

76. Johnson LA, Jackson DG. Inflammation-induced secretion of CCL21 in lymphatic endothelium is a key regulator of integrin-mediated dendritic cell transmigration. Int Immunol. 2010;22(10):839-849.

77. Vigl B, et al. Tissue inflammation modulates gene expression of lymphatic endothelial cells and dendritic cell migration in a stimulus-dependent manner. Blood. 2011;118(1):205-215

78. Kelley PM, Connor AL, Tempero RM. Lymphatic vessel memory stimulated by recurrent inflammation. Am J Pathol. 2013;182(6):2418-2428.

79. Truman LA, et al. ProxTom lymphatic vessel reporter mice reveal Prox 1 expression in the adrenal medulla, megakaryocytes, and platelets. Am J Pathol. 2012;180(4):1715-1725.

80. Bentley KL, Stranford S, Liao S, Mounzer RM, Ruddle FH, Ruddle NH. High endothelial venule reporter mice to probe regulation of lymph node vasculature. Adv Exp Med Biol. 2011;691:35-44.

81. Martínez-Corral I, Olmeda D, Diéguez-Hurtado R, Tammela T, Alitalo K, Ortega S. In vivo imaging of lymphatic vessels in development, wound healing, inflammation, and tumor metastasis. Proc Natl Acad Sci U S A. 2012;109(16):6223-6228.

82. Mempel TR, Scimone ML, Mora JR, von Andrian UH. In vivo imaging of leukocyte trafficking in blood vessels and tissues. Curr Opin Immunol. 2004; 16(4):406-417.

83. Park J, et al. Combination delivery of TGF- $\beta$ inhibitor and IL-2 by nanoscale liposomal polymeric gels enhances tumour immunotherapy. Nat Mater. 2012;11(10):895-905.

84. Look M, Saltzman WM, Craft J, Fahmy TM. The nanomaterial-dependent modulation of dendritic cells and its potential influence on therapeutic immunosuppression in lupus. Biomaterials. 2014; 35(3):1089-1095

85. Achen MG, Stacker SA. Tumor lymphangiogenesis and metastatic spread-new players begin to emerge. Int J Cancer. 2006;119(8):1755-1760.

86. Jeltsch M, Leppänen VM, Saharinen P, Alitalo K. Receptor tyrosine kinase-mediated angiogenesis. Cold Spring Harb Perspect Biol. 2013;5(9):a009183. 